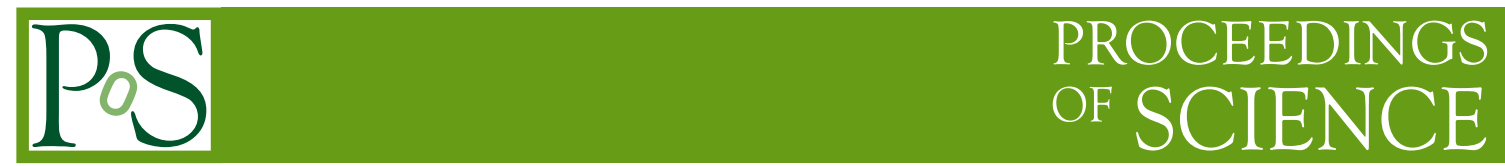

\title{
Keeping our radio windows on the Universe clean
}

\author{
Wim van Driel*广 \\ Observatoire de Paris; CRAF, IUCAF \\ E-mail: wim.vandriel@obspm.fr
}

Keeping the frequency bands used for radio astronomy as free as possible of unwanted Radio Frequency Interference (RFI) is crucial. The aim of Spectrum Management, one of the tools used towards achieving this goal, includes setting regulatory limits on RFI levels emitted by other spectrum users into the radio astronomy frequency bands. This involves discussions with regulatory bodies and other spectrum users at several levels - national, regional and worldwide.

The First MCCT-SKADS Training School

September 23-29 2007

Medicina Bologna, Italy

* Speaker.

$\dagger$ 


\section{Radio Frequency Interference (RFI) - with us from the beginning}

Looking for RFI, he found Astronomy - Karl Jansky (1932): Investigating static for longdistance radio telephone service he found "a faint steady hiss of unknown origin" at a frequency of $20 \mathrm{MHz}(=\lambda 14.5 \mathrm{~m})$, which turned out to be of celestial origin: the Galactic Centre.

Looking for Astronomy, he found RFI - Grote Reber (1940's): While mapping the radio emission from the Milky Way at frequencies of 160 and $480 \mathrm{MHz}$ his data were full of spiky emission due to car ignitions. He found that the RFI in his data was manageable, however: the intermediate time scales of the radio signal from the Milky Way was different from that of the car ignitions and the slowly drifting baselines due to receiver gain instability.

Radio telescopes keep getting larger, but not necessarily more sensitive to RFI: Although radio telescope sensitivity has doubled every 3 years (so far, a factor 100,000 in the 60 years since Grote Reber), and telecommunication keeps getting busier, most RFI enters through far telescope sidelobes (which have not become more sensitive with time), from directions (far) away from where the telescope is pointed, and from (far) outside the frequency band in which the astronomical observations are made.

RFI - how bad can it get: Its negative impact on the quality and reliability of radio astronomical observations can range from mildly inconvenient to smoke coming from the receivers (and astronomers), cf. Fig. 1 - following Cohen (2005):

\section{RFI level: and its effect}

Manageable: much weaker than desired signal

Inconvenient: need to repeat observations

Embarrassing: weak, but leads to fake "detection"

Distressing: multiple failures

Obliterative: pointless to observe further

Destructive: permanent damage to receiver

How can we keep/get RFI out of our data?: Given the many ways in which RFI can get into our data this is a complex situation without a "magic bullet" solution, i.e., no single technique exists that can address all possible scenarios. Methods to avoid and/or minimize the impact of RFI mitigation can be divided into two broad categories: regulatory (spectrum management) and technical (RFI mitigation).

Regulatory methods involve putting regulatory (legal) limits on RFI into radio astronomy frequency bands, and technical methods are aimed at removing RFI signals from radio astronomy observations. Within SKADS both methods are included, involving almost 19 FTE for RFI mitigation studies over a 4 year period, and collaboration towards the establishment of a protected Radio Quiet Zone for the SKA.

As spectrum management, rather than RFI mitigation, is my field of expertise and it was the main topic of my presentation, I will leave a more comprehensive description of technical RFI mitigation techniques to a next SKADS MCCT School. 


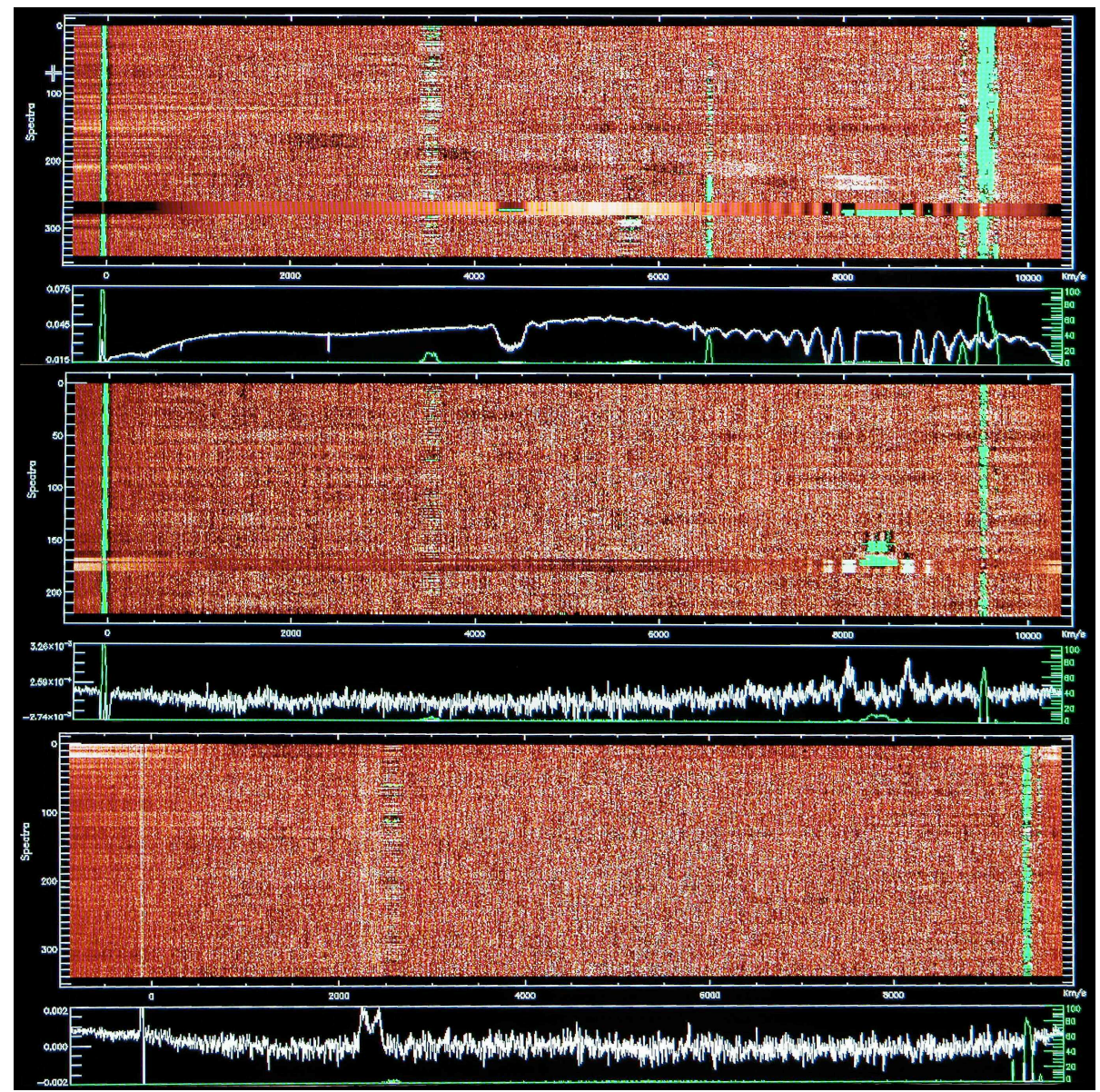

Figure 1: Three levels of RFI in $21 \mathrm{~cm}$ HI line spectra taken with a single-dish radio telescope for a galaxy redshift survey. Each panel shows a waterfall display of the data, with time as vertical axis and radial velocity (in $\mathrm{km} / \mathrm{s}$ ) as horizontal axis; the green data were flagged as RFI and removed from the time-averaged spectrum shown as the white plot underneath each waterfall display: Top panel: inconvenient (RFI too strong during part of the observation, need to repeat observation), middle: embarassing (remaining satellite RFI around $8200 \mathrm{~km} / \mathrm{s}$ mimics a double-horned galaxy HI profile, and bottom: manageable (the RFI on the flank of the HI profile of a galaxy at $2300 \mathrm{~km} / \mathrm{s}$ is faint enough).

\section{Spectrum Management: regulatory solutions}

The underlying principle of spectrum management is "Prevention is Better than a Cure": putting regulatory limits on RFI that can be emitted into the radio astronomy frequency bands will make it easier to deal with it.

The radio spectrum is a finite, and increasingly precious resource for astronomical research, as well as for other spectrum users - a lot of whom want to use the spectrum to make a lot of money, and for whom there is little economic incentive to implement methods (filters, etc.) that reduce RFI into our bands.

Spectrum management can be defined as the task of accommodating all competing radio services and systems within the finite range of the radio frequency spectrum, e.g., by allocating frequency bands, and defining limits on RFI. 
Regulating the electromagnetic smog: over a hundred new satellites are launched each year, and new applications of radio and electronics keep appearing, while national spectrum management Administrations prefer deregulation and spectrum pricing (what price the radio astronomy bands?). Few other radio services notice the rising levels of radio pollution, as they can usually crank up their transmitting power to stay above the RFI - an option radio astronomy does not have, as we cannot increase the transmission power of our celestial radio sources...

\subsection{Our regulatory workhorse: $\operatorname{Rec} 769$}

Called for short "rec seven-six-nine", its formal name is ITU-R Recommendation RA.769 on "Protection criteria used for radio astronomical measurements". It gives threshold levels for RFI detrimental to radio astronomy observations, i.e., interference that introduces a change in amplitude (voltage) at the output of a receiver equal to $1 / 10$ of the rms noise, in a total-power measurement in other words, RFI that increases the uncertainty of a radio astronomy measurements by $10 \%$, i.e., which reduces the effective integration time by $20 \%$.

The Recommendation gives detrimental RFI threshold levels for both continuum and spectral line measurements with single-dish telescopes (Total Power), linked interferometers (like Westerbork), and VLBI. Active service operators often consider our detrimental RFI levels "unrealistically low", and open to negotiation, but they are not used to detecting faint celestial radio sources. For us radio astronomers, Rec 769 is literally "The Limit", and its levels are non-negotiable - accepting RFI that is $10 \mathrm{~dB}$ above the Rec 769 threshold level means we will loose the use of that band in practice.

\subsection{Spectrum Management problem space}

In Figure 2, which illustrates the complex "problem space" of Spectrum Management, there are three axes (passive services, active services, Administrations) and spheres with three different radii (national, regional, global). This indicates that the practical regulatory issues confronting us have a very wide scope and require interactions at quite different levels - they can vary from dealing with local authorities on a planned mobile phone tower that is too close to a radio observatory to working at a global level towards defining RFI limits on unwanted emissions from satellites.

\section{Active and passive Services, and Administrations:}

In regulatory terms, the Radio Astronomy Service is considered a "passive" (i.e., non-emitting) service, meaning that we do not emit electromagnetic radiation ourselves, but build large radio telescopes in order to detect faint natural radio sources. The active services do emit radiation, and their goals are often orthogonal to our own: the RFI they generate can potentially degrade the quality of radio astronomy observations to levels which are unacceptable to us, but the practical means to avoid this (e.g., through installing extra filters in satellites, which adds to their launch weight and cost) may be considered an "undue burden" by them. The role of the Administrations is to ensure the equitable use of the radio spectrum, which often includes playing the role of an impartial referee between the active and passive radio services. 
Administrations regulate

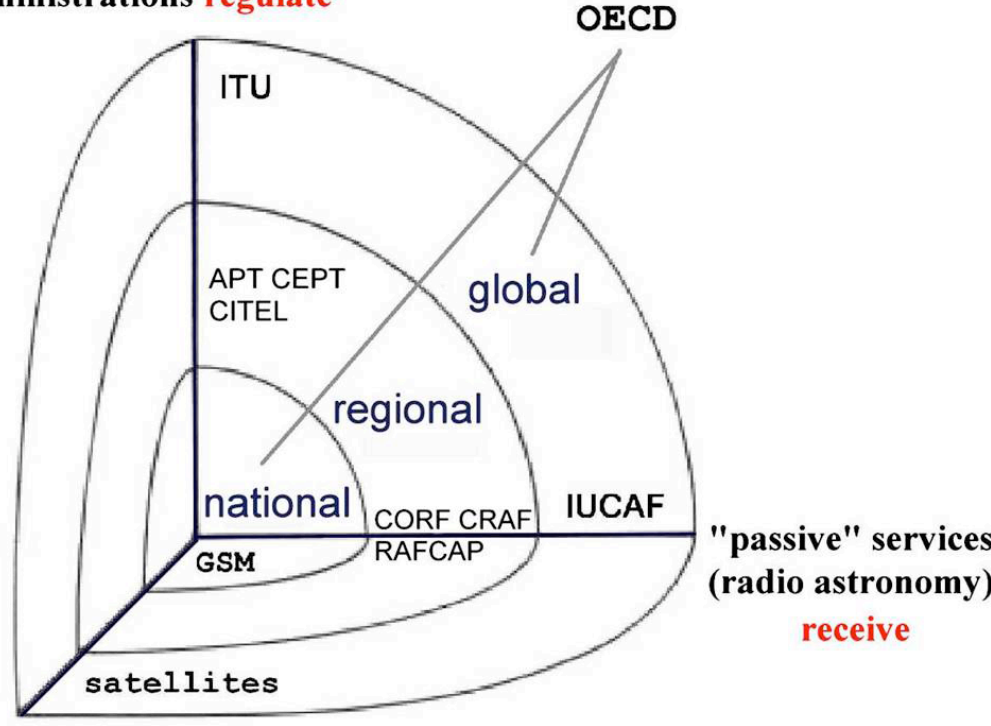

"active" services

emit

Figure 2: The complex "problem space" of spectrum management, with three orthogonal axes (passive services, active services, Administrations) and three spheres of different radii (national, regional, global).

National, regional and global spheres:

Each sovereign state has, in some way or other, its own spectrum management Administration with the mandate to use all means possible to facilitate and regulate (enforce) radiocommunication in that country. Their mandates and terms of reference are usually defined by national telecommunication laws, which also include a national frequency allocation table, which is the national articulation of the ITU Radio Regulations. .

Since radio waves cannot notice national borders, it is imperative that these Administrations coordinate their efforts internationally. The international Administrative cooperation body that coordinates spectrum management at the global level is the International Telecommunication Union, ITU (www.itu.int/net/home/index.aspx).

The global framework for radio spectrum management is provided by the Radio Regulations of the ITU, which have international treaty status and thus are binding for all members of the ITU. They provide rules to national Administrations that allow them to regulate equitable access to the radio spectrum for all entities requiring frequency allocations: telecommunication industry, safety services, aeronautical services, various scientific and hobby uses, etc. The Radio Regulations contain the international Frequency Allocation Table, and, e.g., rules for the use, operation and coordination of frequencies - including limits on RFI into radio astronomy bands.

Between the broad global framework established by the ITU and the detailed frequency planning necessary for national Administrations, there has always been a need for regional coordination. Although, especially from the outside, "Europe" is commonly regarded as equivalent to those 
countries assembled in the European Union, for spectrum management matters Europe covers a considerably larger territory: the 48 countries of the CEPT, the European Conference of Postal and Telecommunications Administrations (www.cept.org).

\section{Radio astronomy spectrum management in Europe}

In spectrum management matters, the European radio astronomy community is represented by CRAF (www.craf.eu), the Expert Committee on Radio Astronomy Frequencies of ESF, the European Science Foundation. CRAF was founded in 1987. Its members represent the radio astronomical observatories of 19 CEPT countries, the European VLBI Network (EVN), the Joint Institute for VLBI in Europe (JIVE) and 3 other multi-national organisations (the European Incoherent Scatter Scientific Association, EISCAT, ESA, and the Institut de Radio Astronomie Millimétrique, IRAM). Together, these observatories cover the entire ITU frequency allocation range, from $13 \mathrm{MHz}$ to 275 GHz.

The European Science Foundation (ESF) acts as a catalyst for the development of science by bringing together leading scientists and funding agencies to debate, plan and implement panEuropean scientific and science policy initiatives. It is the association of 70 major national funding agencies devoted to scientific research in 27 countries and it represents all scientific disciplines.

The role of CRAF is "to keep the frequency bands used by radio astronomers free of interference". To this end it operates both at an administrative and at a technical level: CRAF co-ordinates the relevant representations concerning radio astronomy made to the various national and supranational radio regulatory bodies within Europe, it acts as the European voice in concert with other groups of radio astronomers in discussions within the international bodies that allocate frequencies and it initiates and encourages scientific studies aimed at reducing interference at source and the effects of interference.

Since 1997 CRAF employs a full time pan-European radio astronomy Spectrum Manager. Funding for this position is being provided by the member Institutes or their funding Agencies, and financial support within the sixth and seventh Framework Programme, FP6 and FP7, of the EU is obtained as part of RadioNet, the Radio Astronomy Integrated Infrastructure Initiative (www.radioneteu.org/na/na8.php).

Within the CEPT, CRAF has an observer status, enabling it to participate in its own right in CEPT work at various levels, such as the Working groups FM (Frequency Management) and SE (Spectrum Efficiency), various FM and SE project teams and in the preparation of European Common Positions on issues for the ITU World Radiocommunication Conferences (WRCs), where the ITU Radio Regulations are revised once every 3 to 4 years. CRAF's relation with the European Commission is at present incidental only, as the CEPT handles spectrum management issues within Europe (of which the EU countries are a subset). These ties will need to be intensified, given the views of the EC towards the proliferation of active spectrum applications potentially detrimental for passive services, such as Ultra Wide-Band applications.

At the global level, CRAF is an ITU-R sector member. In general, however, CRAF does not contribute input papers directly to the various ITU-R fora, since it prefers to make its positions know there through input papers from various CEPT fora to ITU bodies, and through collaboration and consultation with IUCAF (www.iucaf.org), the worldwide organisation of radio astronomers 
in spectrum management. At the regional level, with CORF (www7.nationalacademies.org/corf/), representing US radio astronomy, CRAF has an official liaison, which it also hopes to establish with the more recently created RAFCAP (www.atnf.csiro.au/rafcap/), representing radio astronomers in the Asia-Pacific region.

CRAF also has an educational role in making others, particularly active radio spectrum users, aware of the sensitivity and consequent need for protection of the Radio Astronomy Service. Working towards this, CRAF has, e.g., published the CRAF Handbook for Radio Astronomy (3rd ed., 2005) and the CRAF Handbook for Frequency Management (2002), which are made widely available in print and online. Furthermore, CRAF regularly publishes a Newsletter, which is available on its website and distributed in print.

\section{References}

[1] R.J. Cohen, Interference to Radio Astronomy, in Second Summer School in Spectrum Management for Radio Astronomy 2005

[http://www.radionet-eu.org/rnwiki/SpectrumManagementWikiPage].

[2] CRAF, Handbook for Radio Astronomy, European Science Foundation, Strasbourg 2005 [http://www.craf.eu/handbook.htm].

[3] CRAF, Handbook for Frequency Management, European Science Foundation, Strasbourg 2002 [http: //www.craf .eu/hbktm.htm]. 